\title{
Endometrial ossification in a postmenopausal
}

\section{woman}

\author{
M Shimizu, M Nakayama
}

Endometrial ossification is a rare occurrence, ${ }^{1}$ and has also been described as osseous metaplasia of the endometrium, ${ }^{2}$ ectopic intrauterine bone ${ }^{3}$ and heterotopic intrauterine bone formation. ${ }^{4}$ Most patients presenting with endometrial ossification are between 20 and 40 years of age $^{4}$ and the common underlying factors are a history of menstrual irregularities, recurrent abortions, and endometritis. ${ }^{5}$ Endometrial ossification may present as secondary infertility, prolonged unexplained vaginal bleeding, or intrauterine pain. ${ }^{3}$ An erroneous diagnosis of malignant mixed müllerian tumour in the endometrial curettage specimen should be avoided, especially in postmenopausal women. Here, we describe a rare case of endometrial ossification occurring in a postmenopausal woman.

\section{Case report}

In February 1994, a 62 year old woman, gravida 6, para 4, presented to Mizushima Central Hospital because of increased vaginal discharge. Her clinical history revealed a therapeutic abortion and a miscarriage in 1957. She had had four full-term, normal deliveries-in $1951,1955,1959$, and 1961. She ceased menstruating in 1977. Physical and pelvic examination were unremarkable. Transvaginal ultrasound examination revealed a hyperechoic area within the uterine cavity, suggestive of an intrauterine foreign body (fig 1); however, the patient denied the use of an intrauterine contraceptive device. An endometrial curettage was performed and bone-like tissues were removed. The histological examination showed extensive ossification of the endometrium. Decalcified sections showed mature woven bone with neutrophilic infiltration. Foci of bacteria were present. Fragmentation of endometrial glands was also observed (fig 2). There was no evidence of hyperplasia, atypia or malignancy. Two years and six months after treatment with antibiotics, the patient is well and symptom-free.

\section{Discussion}

Endometrial ossification is a rare pathological occurrence, the most common underlying factor of which is a recent history of abortion, ${ }^{6}$ although its pathogenesis remains unclear. Endometrial ossification can be distinguished from retained fetal tissue by the absence of tissue reaction and enchondral ossification. ${ }^{2}$ Endometrial ossification may cause menstrual bleeding, pelvic pain, vaginal discharge, and infertility. ${ }^{3}$ Although our patient had a history of therapeutic abortion and miscarriage, these occurred 37 years previously. Another underly-
Figure 1 Transvaginal ultrasound showing a hyperechoic area within the uterine cavity, suggestive of an intrauterine foreign body. 


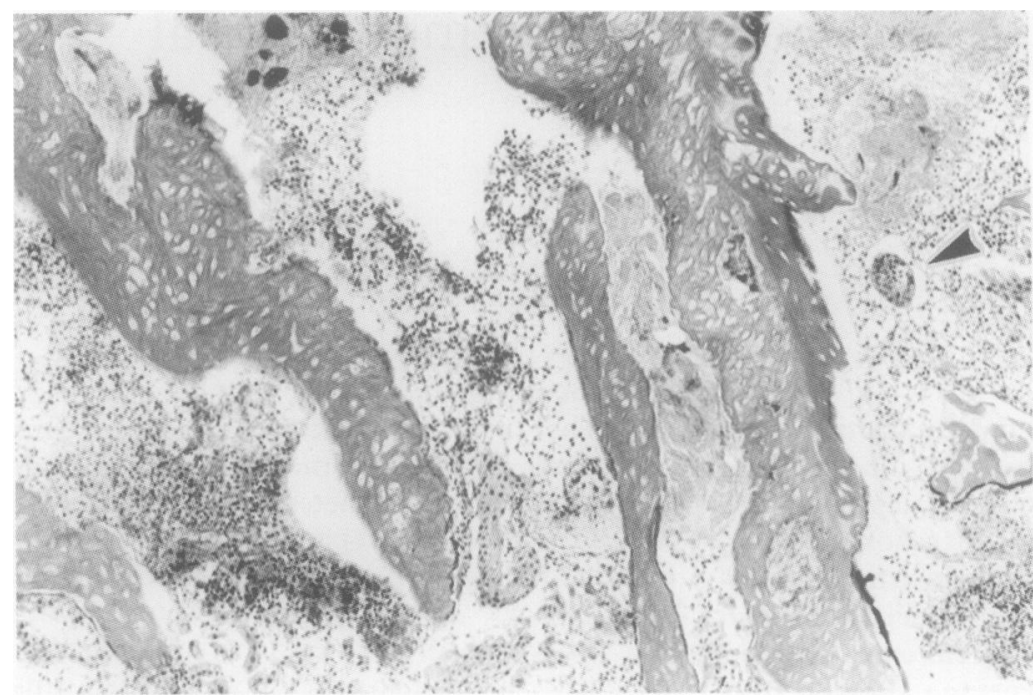

Figure 2 Endometrial curettage revealing the bone tissue and a fragment of an endometrial gland (arrow head) with neutrophilic infiltration.

ing cause of endometrial ossification is endometritis, especially chronic endometritis, as ossification may develop in the old, healing inflammatory tissue. ${ }^{7}$ Prolonged oestrogenic stimulation is thought to be related to the pathogenesis of endometrial ossification, ${ }^{6}$ however, this is unlikely in this patient.

Bone formation in the endometrium is rare, but may be observed in malignant mixed müllerian tumour and teratoma, ${ }^{8}$ which should be considered as a differential diagnosis, ${ }^{9}$ especially in older women. ${ }^{10}$
In summary, endometrial ossification in postmenopausal women is very rare; most women presenting with this condition are between 20 and 40 years of age. Therefore, clinicians should consider the possibility of endometrial ossification as a differential diagnosis of intrauterine foreign body on ultrasound, even in older patients. In addition, pathologists should be aware of this rare entity to avoid a misdiagnosis of malignant mixed müllerian tumour in the endometrial curettage specimen, which may result in unnecessary hysterectomy.

1 Qi L, Qunli S, Yanhua S, Xiaojuan Y. Pregnancy achieved after treatment in a case of endometrial ossification. A case report and literature review. Chin Med f 1995;108:793-5.

2 Acharya U, Pinion SB, Parkin DE, Hamilton MPR. Osseous metaplasia of the endometrium treated by hysteroscopic resection. Br F Obstet Gynaecol 1993;100:391-2.

3 Rodriguez BD, Adamson GD. Hysteroscopic treatment of ectopic intrauterine bone. A case report. $f$ Reprod Med 1993;38:515-20.

4 Taylor PJ, Hamou J, Mencaglia L. Hysteroscopic detection of heterotopic intrauterine bone formation. $f$ Reprod Med 1988;33:337-9.

5 Bhatia NN, Hoshiko MG. Uterine osseous metaplasia Obstet Gynecol 1982;60:256-9.

6 Ceccacci L, Clancy G. Endometrial ossification: report of an additional case. Am $\mathscr{f}$ Obstet Gynecol 1981;141:103-4.

7 Waxman M, Moussouris HF. Endometrial ossification following an abortion. Am f Obstet Gynecol 1978;130:5878.

8 Wetzels LCG, Essed GGM, de Haan J, van de Kar AJF Willebrand D. Endometrial ossification: unilateral manifestation in a septate uterus. Gynecol Obstet Invest 1982;14:4755.

9 Ombelet W. Endometrial ossification, an unusual finding in an infertility clinic. A case report. $f$ Reprod Med 1989;34:303-6.

10 Barwick KW, LiVolsi VA. Malignant mixed müllerian tumors of the uterus. Am $\mathcal{F}$ Surg Pathol 1979;3:125-35.
Department of Pathology, Kawasaki Medical School, Kurashiki, Okayama Japan

M Shimizu

$M$ Hirokawa

T Manabe

Department of Breast and Thyroid Surgery K Shimozuma

H Sonoo

Kawasaki College of Allied Health

Professions

T Harada

Correspondence to: Dr Michio Shimizu, Department of Pathology, Kawasaki Medical School Kurashiki, Okayama 701-01, Japan

Accepted for publication 3 October 1996

\title{
Lithium associated autoimmune thyroiditis
}

\author{
M Shimizu, M Hirokawa, T Manabe, K Shimozuma, H Sonoo, T Harada
}

\begin{abstract}
A case of autoimmune thyroiditis after long term treatment with lithium is described in a 29 year old Japanese woman with manic depression. Positive serum antithyroglobulin and antimicrosomal antibodies, diffuse goitre, and microscopic chronic thyroiditis, as well as the clinical history of long term lithium treatment were suggestive of lithium associated autoimmune thyroiditis. Microscopically, there was a mild degree of interstitial fibrosis and a moderate degree of lymphocytic infiltration. Some areas showed a moderate degree of stromal fibrosis and atrophic thyroid follicles. Lymphoid follicles with germinal centres, disrupted thyroid follicles with lymphocytic infiltration, and Hürthle cells were also observed. The differential diagnosis in
\end{abstract}

patients presenting with these histological features includes painless (silent) thyroiditis, autoimmune thyroiditis and lithium associated autoimmune thyroiditis. A detailed clinical history is essential if the correct diagnosis is to be reached. (f Clin Pathol 1997;50:172-174)

Keywords: thyroid; lithium; autoimmune thyroiditis.

Lithium has been used in the treatment of manic and hypomanic depressive disorders for many years. Long term treatment with lithium is associated with hypothyroidism, euthyroid goitre, and hyperthyroidism. ${ }^{1-6}$ The aetiology of the first two conditions can be explained by the effect of lithium on the thyroid; however, that of hyperthyroidism has been not been explained adequately. Other cases of lithium related thyroid disease have been reported but 